\title{
Fibromyalgia patients have reduced hippocampal volume compared with healthy controls
}

\author{
This article was published in the following Dove Press journal: \\ Journal of Pain Research \\ 30 January 2015 \\ Number of times this article has been viewed
}

\author{
Christina S McCrae' \\ Andrew M O'Shea' \\ Jeff Boissoneault ${ }^{2}$ \\ Karlyn E Vatthauer' \\ Michael E Robinson ${ }^{1,2}$ \\ Roland Staud ${ }^{2,3}$ \\ William M Perlstein ${ }^{4-7}$ \\ Jason G Craggs' \\ 'Department of Clinical and Health \\ Psychology, ${ }^{2}$ Pain Research and \\ Intervention Center of Excellence, \\ ${ }^{3}$ College of Medicine, University \\ of Florida, Gainesville, FL, USA; \\ ${ }^{4}$ McKnight Brain Institute, University \\ of Florida, Gainesville, FL, USA; \\ ${ }^{5}$ Department of Psychiatry, University \\ of Florida, Gainesville, FL, ${ }^{6}$ Malcom \\ Randall Veterans Administration \\ Medical Center, Gainesville, FL, \\ ${ }^{7}$ Rehabilitation Research and \\ Development Brain Research Center \\ of Excellence, Veterans Administration \\ Medical Center, Gainesville, FL, USA
}

Correspondence: Christina S McCrae Department of Health Psychology, University of Missouri, One Hospital Drive, DCI 16.88, Columbia, MO 652I2, USA Tel +l 5738820982

Email mccraec@health.missouri.edu
Objective: Fibromyalgia patients frequently report cognitive abnormalities. As the hippocampus plays an important role in learning and memory, we determined whether individuals with fibromyalgia had smaller hippocampal volume compared with healthy control participants.

Methods: $\mathrm{T}_{1}$-weighted structural magnetic resonance imaging (MRI) scans were acquired from 40 female participants with fibromyalgia and 22 female healthy controls. The volume of the hippocampus was estimated using the software FreeSurfer. An analysis of covariance model controlling for potentially confounding factors of age, whole brain size, MRI signal quality, and Beck Depression Inventory scores were used to determine significant group differences.

Results: Fibromyalgia participants had significantly smaller hippocampi in both left $\left(F[1,56]=4.55, P=0.037, \eta_{\mathrm{p}}^{2}=0.08\right)$ and right hemispheres $\left(F[1,56]=5.89, P=0.019, \eta_{\mathrm{p}}^{2}=0.10\right)$. No significant effect of depression was observed in either left or right hemisphere hippocampal volume ( $P=0.813$ and $P=0.811$, respectively).

Discussion: Potential mechanisms for reduced hippocampal volume in fibromyalgia include abnormal glutamate excitatory neurotransmission and glucocorticoid dysfunction; these factors can lead to neuronal atrophy, through excitotoxicity, and disrupt neurogenesis in the hippocampus. Hippocampal atrophy may play a role in memory and cognitive complaints among fibromyalgia patients.

Keywords: hippocampus, MRI, brain atrophy, pain

\section{Introduction}

Fibromyalgia (FM) is a disorder characterized by chronic, widespread pain in the muscles, ligaments, tendons, and other connective tissues. ${ }^{1}$ Approximately five million people in the United States suffer from FM; annual costs of the disease are estimated around $\$ 6,000$ per person annually. ${ }^{2,3}$ The exact etiology of FM is unknown; ${ }^{4}$ however, evidence of abnormalities in brain structure has been reported. ${ }^{5} \mathrm{FM}$ is currently thought of as a complex disorder involving multiple systems, including both peripheral and central nervous system components.

FM patients have been shown to have reductions in cortical gray matter volumes in numerous areas associated with the pain experience, including the insular and anterior cingulate cortices. ${ }^{6-8}$ However, FM is a disorder characterized by numerous symptoms besides pain, such as sleep disturbance, mood disorders, and cognitive dysfunction. Morphometric changes in FM have been described in areas outside the typically reported cortical "pain regions", such as reduced gray matter in the amygdala. ${ }^{6,9}$

Changes to limbic morphometry in regions such as the cingulate, amygdala, thalamus, and the parahippocampal gyrus, have previously been reported in patients 
with FM. ${ }^{6-10}$ Although reports of alterations in hippocampal volume among chronic pain conditions are sparse, a pivotal role for the hippocampus in FM has been proposed. ${ }^{11}$ Using proton magnetic resonance imaging (MRI) spectroscopy, Wood et al found abnormal ratios of $\mathrm{N}$-acetylaspartate to creatine in the right hippocampus of FM patients compared with healthy controls; significant correlations were observed between these abnormalities and FM symptom severity. ${ }^{12}$ While the cause for such changes is currently unknown, excessive excitatory neurotransmission in the hippocampus may occur in FM, which can lead to potential dysfunction and atrophy.

Hippocampal atrophy resulting from FM may, in turn, worsen or exacerbate FM symptomatology. For example, FM patients often report a subjective worsening of cognitive function characterized by short-term memory problems (ie, the "fibro fog"). ${ }^{13}$ Furthermore, the hippocampal atrophy and dysfunction may "feed forward", resulting in more severe pain, discomfort, and anxiety in FM patients, due to its central role in limbic circuits and pain modulation networks. ${ }^{14}$

While having a strong theoretical backing, conflicting empirical evidence regarding hippocampal volumes in chronic pain conditions exists in the literature. Mutso et al ${ }^{15}$ found significant bilateral decreases in hippocampal volumes, compared with healthy controls, in complex regional pain syndrome (CRPS) patients and chronic back pain patients but not in osteoarthritis patients. However, Maleki et al ${ }^{16}$ found no significant reduction in hippocampal volumes among high-frequency migraine patients relative to controls. Zimmerman et al $^{17}$ reported trend-level $(P=0.06)$ reductions in hippocampal volume in elderly individuals with chronic pain symptoms. While, Lutz et al ${ }^{18}$ reported highly significant $(P<0.001)$ reductions in bilateral hippocampal volume among FM patients; however, depressive symptomology was not explicitly controlled. Similar reductions in cortical gray matter density have been reported in the parahippocampal gyri among FM patients. ${ }^{7,10}$

In order to test the hypothesis that FM patients would have decreased hippocampal volume compared with an asymptomatic control sample, an in vivo proxy of hippocampal gray matter volume was used. We also explored whether any reductions in hippocampal volume were related to severity and chronicity of FM symptomology.

\section{Materials and methods Participants}

A total of 22 healthy control $(\mathrm{HC})$ females (mean age $=43.43$ years) and $40 \mathrm{FM}$ females (mean age $=50.17$ years) were recruited from the University of Florida and surrounding community through radio, newspaper, and television advertisements. HC participants were excluded if they had a history of painful neuropathies, diabetes, or cancer and/or were currently regularly taking the following medications: nonsteroidal antiinflammatory drugs (NSAIDs), antidepressants, antihistamines, any analgesics, anticonvulsants, muscle relaxants, or benzodiazepines. FM participants were sourced from a larger clinical trial investigating comorbid FM and insomnia. Inclusion criteria for FM were: a) age 18 years or older; b) individual reports widespread pain; c) FM confirmed by tender point test, using guidelines established by the American College of Rheumatology (with application of $4 \mathrm{~kg}$ force, participant reported pain in at least eleven of 18 points, including points in all four body quadrants); ${ }^{19} \mathrm{~d}$ ) no prescribed or over-the-counter sleep medication for at least 1 month, or stabilized on medication for at least 6 months; and e) able to read and understand English.

Exclusion criteria were: a) sleep disorder other than insomnia (eg, sleep apnea or periodic limb movement disorder), assessed through structured interview and single-night ambulatory monitoring (disqualified if Apnea-Hypopnea Index or myoclonus arousals greater than $15 /$ hour, or between 10-15/hour with oxygen saturation below $88 \%$ ); b) bipolar disorder or seizure disorder; c) significant medical (eg, cancer) or neurological (eg, dementia) disorder; d) severe untreated psychopathology (eg, schizophrenia, substance abuse); e) cognitive impairment based on a Mini-Mental State Examination score lower than 24 (ninth grade education or higher) or lower than 18 (less than ninth grade education). ${ }^{20}$ The University of Florida Institutional Review Board approved all study procedures. All participants provided written informed consent prior to any study procedures.

\section{Neuroimaging acquisition}

$\mathrm{T}_{1}$-weighted structural MRI scans were acquired from two in-house 3 Tesla (3T) scanners. For FM participants, a Philips Achieva 3.0T 2-Series scanner (Philips Electronics, Amsterdam, The Netherlands) with a Philips eightchannel head coil was used to obtain the structural images. The parameters for each $\mathrm{T}_{1}$-weighted structural scan were set as follows: 180 slices were acquired in the sagittal plane. Fast field echo (FFE) imaging sequence (flip angle $[\mathrm{FA}]=8^{\circ}$; field of view $[\mathrm{FOV}]=240 \mathrm{~mm} \times 240 \mathrm{~mm} \times 180$ $\mathrm{mm}$; matrix $=240 \mathrm{~mm} \times 240 \mathrm{~mm}$; voxel size $=1 \mathrm{~mm} \times 1 \mathrm{~mm}$ $\times 1 \mathrm{~mm}$; slice gap $=0 \mathrm{~mm}$; actual $\mathrm{TR} / \mathrm{TE}=8.1 / 3.7 \mathrm{~ms}$ ) was used. Scans lasted $\sim 6$ minutes (377 seconds). For HC participants, a Siemens MAGNETOM Allegra ${ }^{\text {TM }}$ 3.0T scanner (Siemens AG, Munich, Germany) with an eight-channel head coil was used to obtain the structural images. A $T_{1}$-weighted 
magnetization-prepared rapid gradient-echo (MP-RAGE) protocol $\left(1281 \mathrm{~mm}\right.$ axial slices; $\mathrm{FA}=8^{\circ}$; FOV $=240 \mathrm{~mm}$; matrix $=512 \mathrm{~mm} \times 512 \mathrm{~mm}$; TR $=2,000 \mathrm{~ms}$; TE $=4.13 \mathrm{~ms}$ ) was used. The two scanners used for data collection did not differ significantly in anatomic signal-to-noise ratio (SNR) or white matter signal intensity, which are two important factors for quality image segmentation.

\section{Neuroimaging processing}

To measure hippocampal volume, the automated subcortical segmentation stream in FreeSurfer V.5.1.0 was used. The software uses Bayesian inference methods relying on prior anatomical probabilities in a labeled data set, along with a priori known $T_{1}$ intensity characteristics of subcortical regions, as well as $T_{1}$ intensity information from the scan being processed, in order to label discrete regions. ${ }^{21}$ Previous research has shown this automated procedure produces accurate and reliable results, while taking a fraction of the time of the gold standard of manual segmentation. ${ }^{21,22}$ This makes automated segmentation well suited for large samples.

\section{Statistical analyses}

Several factors of noninterest to this study, such as participant age, total brain size, and signal quality of MRI, have been shown to be associated with differences in estimated hippocampal volume. Although there were no significant differences in the three aforementioned variables between the FM and healthy controls in our sample, we decided to conservatively control them in our analysis, to minimize variance explained by factors other than differences in clinical condition. Differences in gray matter between FM and healthy individuals have previously been linked to the presence of affective disorders, such as major depression; ${ }^{23}$ in order to control for these effects, Beck Depression Inventory-II (BDI) scores were included as a covariate in the analysis. ${ }^{24}$ Missing BDI data for six HC participants were imputed with a random score from the range of HC scores; this method of missing data replacement results in less underestimation of standard errors than mean replacement ("hot deck imputation"). ${ }^{25}$ In addition to controlling for BDI, the main effect of BDI scores on hippocampal volume was explored to examine the possible differential effects of depression and FM.

Two analyses of covariance (ANCOVAs) were run using SPSS v20, with dependent measures of left and right hippocampal volume respectively, a fixed factor of diagnosis, and covariates of BDI, age, whole brain gray matter volume, and anatomic SNR. Whole brain gray matter volume was used as a proxy for brain size, instead of intracranial volume, because whole brain gray matter volume had a stronger relationship to hippocampal volume in our sample ( $r=0.636$ for whole brain gray matter and hippocampal volume relationship compared with $r=0.12$ for total intracranial volume and hippocampal volume relationship).

In order to examine the relationship between FM symptomology severity and hippocampal volume, measures of self-reported sleep quality (ratio of total sleep time to total time in bed, as reported in daily sleep diaries) and Pain Disability Index questionnaire ${ }^{26}$ scores were correlated with hippocampal volume. Measures of sleep quality and pain disability were not collected in the $\mathrm{HC}$ sample due to the data being sourced from separate studies. To examine the relationship between FM chronicity and hippocampal volume, normalized symptom duration (years since symptom onset/ participant's age) was correlated with hippocampal volume. Symptom duration was not compared in HC participants, as it is not applicable. All correlation tests were conducted using semipartial correlations that controlled for the effects of age, total gray matter volume, SNR, and BDI on hippocampal volume, while leaving dependent variables unadjusted. ${ }^{27}$

\section{Results \\ Demographics}

Participants' demographic information is presented in Table 1.

\section{Main effect of group}

ANCOVA indicated FM participants had significantly smaller hippocampi in both left $(F[1,56]=4.55, P=0.037)$ and right hemispheres $(F[1,56]=5.89, P=0.019)$. Larger volume differences were observed in the right hippocampus $\left(\eta_{\mathrm{p}}^{2}=0.095\right)$ compared with the left hippocampus $\left(\eta_{\mathrm{p}}^{2}=0.075\right)$ (Figure 1).

\section{Depressive symptomatology}

As anticipated, significant differences in depression level were observed between FM and HC groups, with FM

Table I Demographic information

\begin{tabular}{|c|c|c|c|c|c|c|}
\hline Group & $\mathbf{N}$ & $\begin{array}{l}\text { Age in } \\
\text { years }\end{array}$ & BDI & SE & PDI & $\begin{array}{l}\text { Symptom } \\
\text { duration } \\
\text { (years) }\end{array}$ \\
\hline $\mathrm{HC}$ & 22 & $\begin{array}{l}43.4 \\
(11.3)\end{array}$ & $\begin{array}{l}3.0 \\
(3.0)\end{array}$ & NA & NA & NA \\
\hline FM & 40 & $\begin{array}{l}51.0 \\
(13.5)\end{array}$ & $\begin{array}{l}14.5 \\
(10.2)\end{array}$ & $\begin{array}{l}79.5 \\
(10.4)\end{array}$ & $\begin{array}{l}30.8 \\
(15.7)\end{array}$ & $\begin{array}{l}11.9 \\
(9.9)\end{array}$ \\
\hline
\end{tabular}

Notes: Values in parentheses are standard deviation. NA indicates data was not collected for the measure.

Abbreviations: $\mathrm{BDI}$, Beck Depression Inventory; FM, fibromyalgia; HC, healthy control; PDI, Pain Disability Index; SE, sleep efficiency. 
A

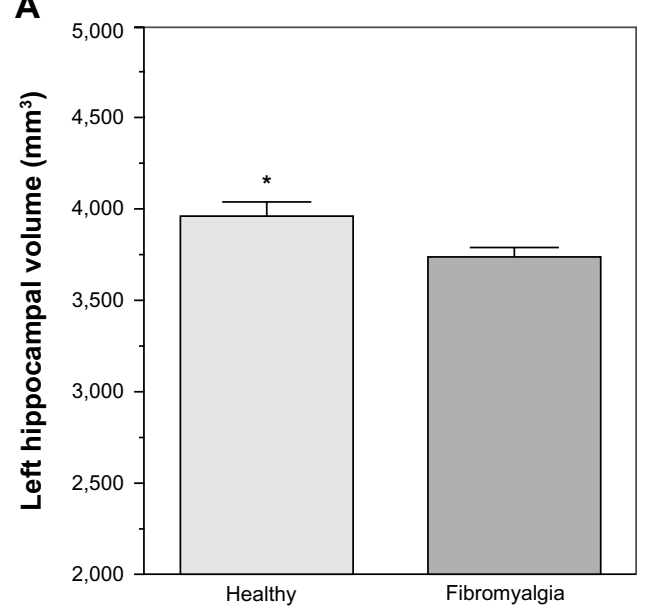

B

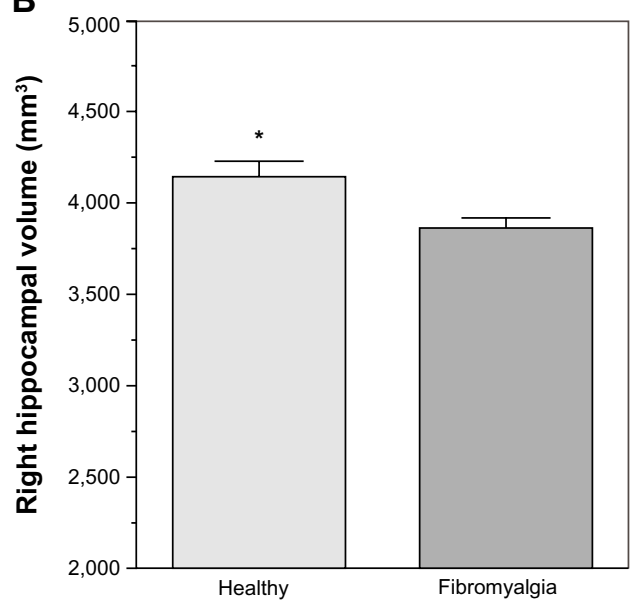

Figure I Estimated left (A) and right (B) hippocampal volume for fibromyalgia and healthy control groups.

Notes: Estimated marginal means. Error bars represent + I standard error of mean. $* P<0.05$.

participants having higher BDI scores $(t[60]=6.58, P<0.001$, Cohen's $d=1.52$ ). However, no significant effect of BDI on hippocampal volume was observed in either hemisphere (left hemisphere: $F[1,56]=0.06, P=0.813$; right hemisphere: $F[1,56]=0.06, P=0.811)$.

\section{Sleep efficiency and hippocampal volume}

No significant relationships were observed between sleep efficiency and hippocampal volume, in either hemisphere in FM participants, using Pearson correlations (left hemisphere: $r=0.05, P=0.796$; right hemisphere $r=0.17, P=0.337$ ).

\section{Pain disability and hippocampal volume}

No significant relationships were observed between Pain Disability Index scores and hippocampal volume, in either hemisphere in FM participants, using Pearson correlations (left hemisphere: $r=-0.17, P=0.351$; right hemisphere $r=-0.05, P=0.788)$.

\section{FM chronicity and hippocampal volume}

No significant relationships were observed between FM chronicity and hippocampal volume, using Pearson correlations (left hemisphere: $r=0.23, P=0.187$; right hemisphere $r=0.22, P=0.209$ ).

\section{Discussion}

While controlling for potentially confounding factors, participants with FM showed reduced hippocampal volume bilaterally. These findings are in line with previous research showing reduced hippocampal volumes in FM. ${ }^{18}$ However, to the authors' knowledge, this is the first report describing reduced hippocampal volumes in FM while explicitly controlling for comorbid depression. Although patients with FM have increased incidence of depression and patients with major depressive disorder have been shown to have smaller hippocampi, ${ }^{28}$ we did not find a significant main effect of depression in our study.

No relationships between FM chronicity, sleep efficiency, or Pain Disability Index and hippocampal volume were observed in our sample. Kuchinad et $\mathrm{al}^{7}$ have described a significant relationship between duration of FM and gray matter deficits. However, other authors have reported no such relationship. ${ }^{10,29}$ Heterogeneity among modestly sized samples, discrepancy in how chronicity is defined (ie, years since symptom onset or years since diagnosis of FM), and inaccurate recall of events that are sometimes decades in the past may explain some of the inconsistency in the literature. Furthermore, we hypothesized participants with more severe symptoms (ie, lower quality and quantity of sleep and greater disability from pain) would show greater reductions in hippocampal volume. However, no such relationship was apparent. It is possible that symptom severity along other dimensions (ie, memory and cognitive function) may be more associated with hippocampal volume, due to the extensive role of the hippocampus in memory and cognition.

\section{Potential mechanisms}

\section{for hippocampal atrophy}

Mechanisms of hippocampal atrophy in FM patients need to be explored further. One potential mechanism includes a dysfunctional balance between excitatory glutamate and inhibitory gamma-aminobutyric acid (GABA) neurotransmission. FM patients have been shown to have elevated levels of excitatory amino acids, such as glutamate. ${ }^{30}$ Clauw et al ${ }^{30}$ posited that 
abnormally high glutamate neurotransmission is associated with hyperalgesia and allodynia. Drugs that attempt to restore the balance between excitatory and inhibitory neurotransmission, via combinations of N-methyl-D-aspartate (NMDA) glutamate antagonism and GABA agonism, have been tested for efficacy in FM (for eg, sodium oxybate and ketamine). ${ }^{31,32}$ Restoring the balance in inhibitory/excitatory neurotransmission appears crucial in limiting hippocampal atrophy, as NMDA excitotoxicity is a prominent factor in animal models of hippocampal neuronal death. ${ }^{33}$

Prolonged or traumatic stress represents another potential mechanism for hippocampal volume deficits in FM. Clinically FM often presents after a biological stressor, such as infection, or a psychosocial stressor, such as a major life event, or combinations of both. ${ }^{34} \mathrm{FM}$ patients have been found to have abnormalities in endogenous glucocorticoid and hypothalamicpituitary-adrenal axis function, two important mediators of the biological stress response. ${ }^{35,36}$ Prolonged stress has been associated with hippocampal atrophy in animal models as well as human neuroimaging studies of posttraumatic stress disorder patients. ${ }^{37,38}$ Some authors have suggested hippocampal volume deficits are a predisposing factor to maladaptive stress response, ${ }^{39}$ while others posit hippocampal atrophy is a consequence of prolonged or traumatic stress. ${ }^{40}$

\section{Limitations and future directions}

Longitudinal studies are needed to clarify the time course of hippocampal atrophy; due to the cross-sectional nature of this study it is unknown whether hippocampal abnormalities precede FM, are a consequence of the disorder, or both. Furthermore, potential neuroplasticity of the hippocampus resulting from treatment is an unknown and exciting area for future research. Experimental designs that manipulate potential mechanisms of hippocampal atrophy (such as behavioral interventions promoting healthy stress responses or pharmacologic interventions targeting potentially excessive glutamatergic neurotransmission) in a FM sample would provide the evidence needed for causal inference. Additionally, technological advances in the acquisition and processing of neuroimaging data may provide new insights (for example, the subdivision of hippocampal structures into functionally distinct subfields, using high resolution structural MRI). ${ }^{41}$

\section{Conclusion}

FM participants show reduced hippocampal volumes when compared with sex-matched healthy controls. These volumetric deficits were not explained by differences in age, duration of illness, total brain size, or depressive symptomology.
Further research on hippocampal structure and function in fibromyalgia is justified.

\section{Acknowledgments}

The project described was supported by award numbers R01AR055160 and R01AR055160-S1 American Recovery and Reinvestment Act Supplement from the National Institute of Arthritis and Musculoskeletal and Skin Diseases.

\section{Disclosure}

The authors report no conflicts of interest in this work.

\section{References}

1. McBeth J, Mulvey MR. Fibromyalgia: mechanisms and potential impact of the ACR 2010 classification criteria. Nat Rev Rheumatol. 2012;8(2):108-116.

2. Lawrence RC, Felson DT, Helmick CG, et al; National Arthritis Data Workgroup. Estimates of the prevalence of arthritis and other rheumatic conditions in the United States. Part II. Arthritis Rheum. 2008;58(1):26-35.

3. Wolfe F, Anderson J, Harkness D, et al. A prospective, longitudinal, multicenter study of service utilization and costs in fibromyalgia. Arthritis Rheum. 1997;40(9):1560-1570.

4. Gormsen L, Rosenberg R, Bach FW, Jensen TS. Depression, anxiety, health-related quality of life and pain in patients with chronic fibromyalgia and neuropathic pain. Eur J Pain. 2010;14(2):127. e1-127. e8.

5. Schweinhardt P, Sauro KM, Bushnell MC. Fibromyalgia: a disorder of the brain? Neuroscientist. 2008;14(5):415-421.

6. Burgmer M, Gaubitz M, Konrad C, et al. Decreased gray matter volumes in the cingulo-frontal cortex and the amygdala in patients with fibromyalgia. Psychosom Med. 2009;71(5):566-573.

7. Kuchinad A, Schweinhardt P, Seminowicz DA, Wood PB, Chizh BA, Bushnell MC. Accelerated brain gray matter loss in fibromyalgia patients: premature aging of the brain? J Neurosci. 2007;27(15):4004-4007.

8. Robinson ME, Craggs JG, Price DD, Perlstein WM, Staud R. Gray matter volumes of pain-related brain areas are decreased in fibromyalgia syndrome. J Pain. 2011;12(4):436-443.

9. Jensen KB, Srinivasan P, Spaeth R, et al. Overlapping structural and functional brain changes in patients with long-term exposure to fibromyalgia pain. Arthritis Rheum. 2013;65(12):3293-3303.

10. Wood PB, Glabus MF, Simpson R, Patterson JC. Changes in gray matter density in fibromyalgia: correlation with dopamine metabolism.J Pain. 2009;10(6):609-618.

11. Wood PB. Fibromyalgia syndrome: a central role for the hippocampus-A theoretical construct. J Musculoskeletal Pain. 2004;12(1):19-26.

12. Wood PB, Ledbetter CR, Glabus MF, Broadwell LK, Patterson JC. Hippocampal metabolite abnormalities in fibromyalgia: correlation with clinical features. J Pain. 2009;10(1):47-52.

13. Ambrose KR, Gracely RH, Glass JM. Fibromyalgia dyscognition: concepts and issues. Reumatismo. 2012;64(4):206-215.

14. Ploghaus A, Narain C, Beckmann CF, et al. Exacerbation of pain by anxiety is associated with activity in a hippocampal network. J Neurosci. 2001;21(24):9896-9903.

15. Mutso AA, Radzicki D, Baliki MN, et al. Abnormalities in hippocampal functioning with persistent pain. $J$ Neurosci. 2012;32(17): $5747-5756$.

16. Maleki N, Becerra L, Brawn J, McEwen B, Burstein R, Borsook D. Common hippocampal structural and functional changes in migraine. Brain Struct Funct. 2013;218(4):903-912.

17. Zimmerman ME, Pan JW, Hetherington HP, Lipton ML, Baigi K, Lipton RB. Hippocampal correlates of pain in healthy elderly adults: a pilot study. Neurology. 2009;73(19):1567-1570. 
18. Lutz J, Jäger L, de Quervain D, et al. White and gray matter abnormalities in the brain of patients with fibromyalgia: a diffusion-tensor and volumetric imaging study. Arthritis Rheum. 2008;58(12):3960-3969.

19. Wolfe F, Smythe HA, Yunus MB, et al. The American College of Rheumatology 1990 criteria for the classification of fibromyalgia. Report of the Multicenter Criteria Committee. Arthritis Rheum. 1990;33(2):160-172.

20. Murden RA, McRae TD, Kaner S, Bucknam ME. Mini-Mental State Exam scores vary with education in blacks and whites. J Am Geriatr Soc. 1991;39(2):149-155.

21. Fischl B, Salat DH, Busa E, et al. Whole brain segmentation: automated labeling of neuroanatomical structures in the human brain. Neuron. 2002;33(3):341-355.

22. Jovicich J, Czanner S, Han X, et al. MRI-derived measurements of human subcortical, ventricular and intracranial brain volumes: Reliability effects of scan sessions, acquisition sequences, data analyses, scanner upgrade, scanner vendors and field strengths. Neuroimage. 2009;46(1):177-192.

23. Hsu MC, Harris RE, Sundgren PC, et al. No consistent difference in gray matter volume between individuals with fibromyalgia and agematched healthy subjects when controlling for affective disorder. Pain. 2009; 143(3):262-267.

24. Beck AT, Steer RA, Ball R, Ranieri W. Comparison of Beck Depression Inventories-IA and -II in psychiatric outpatients. J Pers Assess. 1996; 67(3):588-597.

25. Andridge RR, Little RJ. A review of hot deck imputation for survey non-response. Int Stat Rev. 2010;78(1):40-64.

26. Tait RC, Pollard CA, Margolis RB, Duckro PN, Krause SJ. The Pain Disability Index - psychometric and validity data. Arch Phys Med Rehab. 1987;68(7):438-441.

27. Field A. Discovering Statistics Using SPSS. 3rd ed. London: Sage Publications; 2009.

28. MacQueen GM, Campbell S, McEwen BS, et al. Course of illness, hippocampal function, and hippocampal volume in major depression. Proc Natl Acad Sci U S A. 2003;100(3):1387-1392.

29. Schmidt-Wilcke T, Luerding R, Weigand T, et al. Striatal grey matter increase in patients suffering from fibromyalgia - a voxel-based morphometry study. Pain. 2007;132 Suppl 1:S109-S116.
30. Clauw DJ, Arnold LM, McCarberg BH; FibroCollaborative. The science of fibromyalgia. Mayo Clin Proc. 2011;86(9):907-911.

31. Russell IJ, Holman AJ, Swick TJ, Alvarez-Horine S, Wang YG, Guinta D; Sodium Oxybate 06-008 FM Study Group. Sodium oxybate reduces pain, fatigue, and sleep disturbance and improves functionality in fibromyalgia: results from a 14-week, randomized, double-blind, placebo-controlled study. Pain. 2011;152(5):1007-1017.

32. Graven-Nielsen T, Aspegren Kendall S, Henriksson KG, et al. Ketamine reduces muscle pain, temporal summation, and referred pain in fibromyalgia patients. Pain. 2000;85(3):483-491.

33. McEwen BS. Possible mechanisms for atrophy of the human hippocampus. Mol Psychiatr. 1997;2(3):255-262.

34. Van Houdenhove B, Egle UT. Fibromyalgia: a stress disorder? Piecing the biopsychosocial puzzle together. Psychother Psychosom. 2004;73(5):267-275.

35. Griep EN, Boersma JW, de Kloet ER. Altered reactivity of the hypothalamic-pituitary-adrenal axis in the primary fibromyalgia syndrome. J Rheumatol. 1993;20(3):469-474.

36. Adler GK, Kinsley BT, Hurwitz S, Mossey CJ, Goldenberg DL. Reduced hypothalamic-pituitary and sympathoadrenal responses to hypoglycemia in women with fibromyalgia syndrome. Am J Med. 1999;106(5):534-543.

37. McEwen BS. Stress and hippocampal plasticity. Annu Rev Neurosci. 1999;22:105-122.

38. Bremner JD, Randall P, Scott TM, et al. MRI-based measurement of hippocampal volume in patients with combat-related posttraumatic stress disorder. Am J Psychiatry. 1995;152(7):973-981.

39. Gilbertson MW, Shenton ME, Ciszewski A, et al. Smaller hippocampal volume predicts pathologic vulnerability to psychological trauma. Nat Neurosci. 2002;5(11):1242-1247.

40. Lee T, Jarome T, Li SJ, Kim JJ, Helmstetter FJ. Chronic stress selectively reduces hippocampal volume in rats: a longitudinal magnetic resonance imaging study. Neuroreport. 2009;20(17):1554-1558.

41. Van Leemput K, Bakkour A, Benner T, et al. Automated segmentation of hippocampal subfields from ultra-high resolution in vivo MRI. Hippocampus. 2009;19(6):549-557.
Journal of Pain Research

\section{Publish your work in this journal}

The Journal of Pain Research is an international, peer-reviewed, open access, online journal that welcomes laboratory and clinical findings in the fields of pain research and the prevention and management of pain. Original research, reviews, symposium reports, hypothesis formation and commentaries are all considered for publication.

\section{Dovepress}

The manuscript management system is completely online and includes a very quick and fair peer-review system, which is all easy to use. Visit http://www.dovepress.com/testimonials.php to read real quotes from published authors. 